\title{
Modelo educativo y desafíos en la formación docente
}

\section{Atipaana yachachi amawta yachachi manatinkuninkunawan}

Pedro Barrientos Gutiérrez*

\section{Resumen}

La formación docente bajo la visión mecanicista y fragmentaria de la educación está centrada en la transmisión de información científica académica, realizadas bajo la pedagogía de cumplimiento de contenidos programados y los principios de la educación de calidad. Esta práctica en la formación profesional de la educación genera limitaciones en el desempeño de la práctica educativa. Sin embargo, las demandas sociales actuales exige la implementación de un modelo educativo que responda a una visión holística en la formación humana y profesional del docente con práctica de pedagogía del ejemplo en base a los fundamentos de la integridad y calidad educativa.

\author{
Palabras clave \\ Modelo educativo, \\ formación integral, \\ formación humana \\ y profesional.
}

\section{Shuukukuna limana: \\ Atipaana yachachi, llapanta yachachi, nunakay yachachi yachayninwan.}

Presentado: 18 de agosto de 2017 Corregido 18 de marzo de 2018 Aprobado 18 de abril de 2018.

* Filiación: Universidad Nacional del Centro del Perú.

\footnotetext{
Datos del autor

Pedro Barrientos Gutiérrez. Peruano. Investigador y docente universitario. Doctor en Ciencias de la Educación por la Universidad Nacional Hermilio Valdizán de Huánuco. Magister en Administración de la Educación por la Universidad de Lima. Especialista en Currículo y Supervisión Educativa por la Universidad Nacional Mayor de San Marcos. Especialista en Formación Magisterial por la Pontificia Universidad Católica del Perú. Correo: pebargut@yahoo.es ORCID: https://orcid.org/0000-0003-0390-6206
} 


\section{Educational Model and Challenges in Teacher Training}

\begin{abstract}
Teacher training under the mechanistic and fragmented vision of education is focused on the transmission of academic scientific information, carried out under the pedagogy of fulfillment of programmed content and principles of quality education. This practice in the professional training of education creates limitations in the performance of educational practice. However, the current social demands requires the implementation of an educational model that responds to a holistic human and professional training of the teaching practice of pedagogy of example based on the foundations of integrity and quality educational.
\end{abstract}

\section{Keywords}

Educational model, comprehensive training, professional and human formation.

\section{Modelo educativo e desafios na formação docente}

\begin{abstract}
Resumo
A formação docente desde a visão mecanicista e fragmentada da educação está centrada na transmissão de informação científica acadêmica, realizada sob a pedagogia da realização de conteúdos programados e os princípios da educação de qualidade. Essa prática na formação profissional da educação gera limitações no desempenho da prática educativa. No entanto, as demandas sociais atuais exigem a implementação de um modelo educacional que responda a uma visão holística na formação humana e profissional do docente, com prática da pedagogia do exemplo baseada nos fundamentos da integridade e qualidade educativa.
\end{abstract}

\section{Palavras-chave:} modelo educativo, formação integral, formação profissional e humana. 


\section{A modo de introducción en la experiencia de formación docente}

La unidad de la teoría y la práctica es la base epistemológica del conocimiento humano; la teoría sirve para direccionar el camino de la práctica, pero también hay que tener en cuenta que sin práctica no hay teoría. Sin embargo, actualmente la formación profesional del docente está orientado bajo la visión mecanicista y fragmentaria de la educación, centrado sólo en la transmisión de información científica académica en las aulas, realizadas con la práctica de la pedagogía de cumplimiento de los contenidos programados en el sílabo y de acuerdo a los fundamentos de la educación de calidad; esta práctica en la formación profesional de la educación, viene generando limitaciones y dificultades en el desempeño de la práctica pedagógica de los futuros docentes, por lo que las demandas sociales actuales exige la necesidad de implementar un nuevo modelo educativo que responda a una visión holística en la formación humana y profesional del docente con práctica permanente de la pedagogía del ejemplo en base a los fundamentos de la integridad y calidad educativa.

La visión integral en la formación humana y profesional del docente comprende el desarrollo de las dimensiones humanas y los niveles de conciencia en la consolidación permanente de la vocación de educar a los estudiantes con dignidad, respeto, libertad, diálogo, paciencia, compasión, fraternidad, compromiso y responsabilidad social en el ejercicio del desempeño de la práctica pedagógica; es decir, la formación docente sea para aprender a vivir la educación, abandonando la errónea idea de vivir de la educación. Que la tarea de educar deje de ser un trabajo por cumplimiento de funciones y se convierta en una verdadera vocación por ayudar a los demás a encontrar su verdadero talento, compartir sus adversidades, realizar sus sueños y encontrar la felicidad.

Por consiguiente, las instituciones encargadas de la formación docente abandonen la errónea concepción de la educación científica y la investigación científica bajo la filosofía y enfoque pedagógico positivista o neopositivista de la ciencia que conduce a una visión mecanicista y fragmentaria de la educación actual, enmarcados sólo estudiar para concluir la carrera con la preocupación de aprobar las asignaturas del plan de estudios y luego hacer la investigación cuantitativa para obtener un título profesional de docente sólo como compromiso personal y no como compromiso y responsabilidad social que es la investigación en su verdadero sentido. Esta formación docente deja a un lado la formación del ser (saber ser de la persona) y prioriza la formación científica académica del futuro docente, promovidos con un conjunto de asignaturas del plan de estudios evaluados sólo la memorización de los contenidos académicos de alguna manera trasmitidos en las aulas de formación profesional, convirtiéndose así las universidades en fábrica de títulos profesionales.

En un momento de altas contingencias sociales y naturales que vivimos, la naturaleza de la formación docente debe centrar en el desarrollo del saber ser, que es la esencia del ser humano, la síntesis de su desarrollo integral; la interdisciplinariedad y la transdisciplinariedad de una percepción holística, debe ocupar nuestra preocupación en la materialización curricular de formación docente con una clara tendencia hermenéutica-interpretativa de la realidad educativa, dando respuesta a la realidad más sentida de los pueblos del país y del mundo (Barrientos, 2016, p.170).

El tránsito de la modernidad a la transmodernidad en la formación docente exige un nuevo paradigma, que ya no esté basado en la fragmentación de la realidad, sino en una visión in- 
tegral de la realidad. Necesitamos un diseño curricular de formación docente elaborado bajo el modelo educativo multinivel-multidimensión que responda a una visión integral de la educación y el pluralismo epistemológico, esto implica tener una posición que considera que la realidad educativa tiene diversos niveles de profundidad y que cada uno de estos niveles debe ser estudiado con una base epistemológica diferente, en este sentido las diversas epistemologías más que ser contradictorias son complementarias siempre y cuando sean articuladas en un modelo integral de la educación.

\section{Escenarios, tendencias y prospectiva de la formación docente}

Los retos actuales en el desarrollo de una sociedad con ventajas de sostenibilidad y sustentabilidad, calidad de vida y respeto al desarrollo integral de las personas se percibe cada vez inalcanzable en estos tiempos de la globalización internacional y el uso de las tecnologías de información y comunicación de todos los pueblos de la tierra y de la identidad cultural de los mismos, donde las personas, supuestamente de alta preparación académica, vivimos compartiendo una sociedad de alto riesgo para la vida humana, donde cada vez se acrecienta la corrupción, crimen organizada, contaminación ambiental por actividades urbanas, industriales y mineras, incremento de la delincuencia y drogadicción, asistencialismo de instituciones públicas y privadas, insuficiencia de recursos presupuestales del Estado para educación, incremento del trabajo infantil y adolescente, incremento de enfermedades emergentes y re-emergentes (TBC, VIH, otros), contaminación de ríos, lagunas y litoral marino, etc.

Vivimos en un escenario local, nacional e internacional caótico y caotizante, donde a la educación no se promueve en su verdadero sentido ni en la escuela, comunidad y en el hogar, siendo todos ellos, una comunidad de aprendizaje importante en la formación integral del ser humano. Las exigencias del mundo actual como consecuencia del cambio a una época global tienen profundas implicaciones para la educación, ya que nos obliga a reconocer que ya no es posible educar seres humanos con un paradigma científico industrial del siglo XVII para una sociedad sustentable en el siglo XXI.

El mundo de la ciencia y la tecnología es un elemento necesario, pero no suficiente para construir una nueva cultura y una nueva conciencia de naturaleza holista. Ya no es posible reducir la educación a un entrenamiento de la racionalidad instrumental para actuar dentro de una rutina laboral de procesos mecánicos tal como la sociedad científico industrial lo necesitó en su tiempo. Hoy los sistemas educativos, desde educación básica hasta los postgrados, deben convertirse en espacios donde se promueva formación integral, conciencia de interdependencia, cooperación y paz global. Ello exige un nuevo paradigma educativo y un nuevo rol para el educador. En esta nueva cultura, el educador tendrá una visión y misión muy diferente a la de siglos pasados, la educación deberá estimular y desarrollar ampliamente las habilidades y virtudes humanas (Gallegos, 2003).

Walter Peñaloza (2000, p.159), señala que las instituciones de formación docente, tienen que dar respuesta a las exigencias sociales de la época, garantizando la formación integral de los futuros educadores, ya que se trata de una carrera holística, en cuanto que atañe al ser humano y a su pleno desarrollo como persona. Hoy se fragmenta la formación docente, el resultado es la aniquilación del sentido holístico de la carrera, siendo una torcida preparación de los docentes. Este hecho es decisivo para explicar el fracaso de la educación. 
Gallegos (2003) nos dice que la educación ha fracasado porque se ha basado en supuestos falsos sobre la naturaleza del aprendizaje, la inteligencia, la escuela y el ser humano. Los cambios de la época caracterizada por la emergencia de un nuevo paradigma global de nuestra cultura exigen una nueva educación como alternativa real para solucionar creativamente los problemas de la sociedad industrial-científica y la visión del mundo mecanicista que nació en el siglo XVII y todavía domina nuestras vidas y los objetivos de la educación.

Asimismo, el sistema educativo actual es un intento de muchos años por imponerle a los estudiantes en general, el paradigma mecanicista, un paradigma que estimula codicia, competencia, avaricia, individualismo, confrontación, egocentrismo, racismo, indiferencia, simulación, fingimiento, etc., un paradigma que nutre la sombra del ser humano, estimula lo peor de la humanidad y que, en realidad, bajo sus principios de estandarización, calidad y fragmentación impiden el aprendizaje significativo, integral y espiritual de los estudiantes (Gallegos, 2000).

La universidad en la formación docente cumple un rol trascendental para el desarrollo local, nacional e internacional en la construcción de una visión de futuro de sociedad basada en la prospectiva para gestar una sociedad democrática, justa, intercultural, pacífica, ecoeficiente, sostenible y solidarios. En este contexto, las tendencias actuales en la educación superior en formación docente deben ser direccionados bajo el enfoque de la educación holística, siendo para ello el modelo educativo integral con perspectiva multinivel - multidimensión.

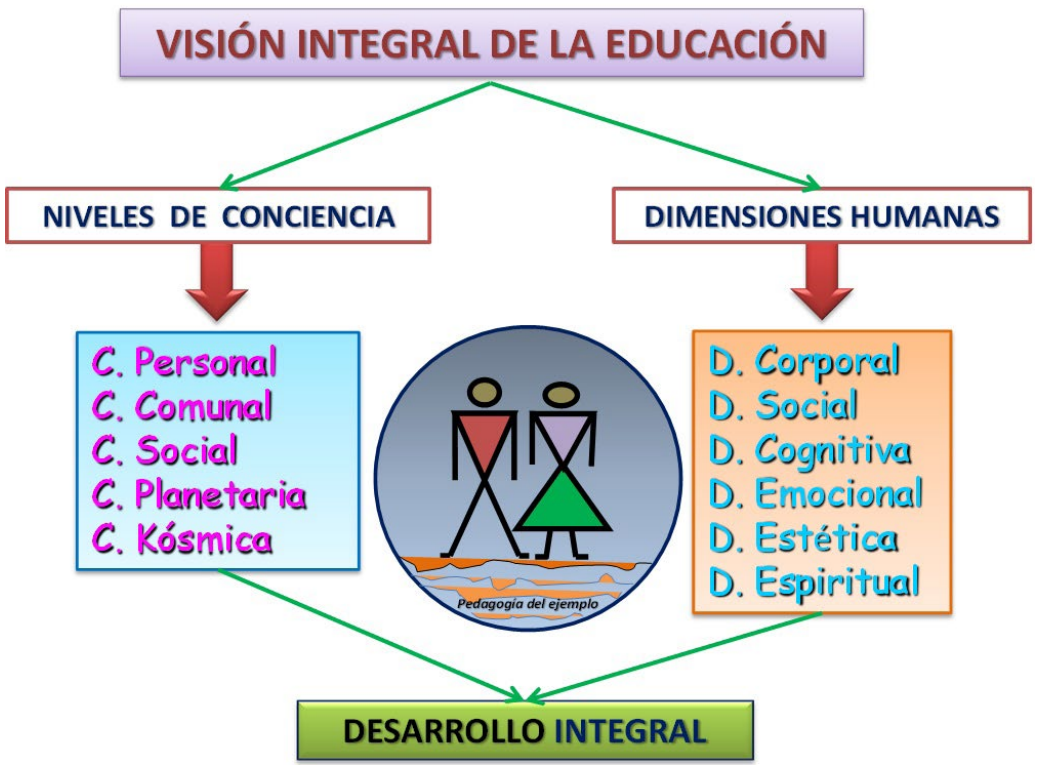

Según el enfoque holístico de la educación, la formación docente debe ser bajo una perspectiva integral de la educación, siendo una formación centrada en la esencia del ser humano más que una formación en el entrenamiento mecánico para el cumplimiento de las funciones. La esencia en la formación docente es el desarrollo de cualidades creativas, estratégicas, científicas, emocionales, estéticas y valores como la responsabilidad, el respeto, la gratitud, la justicia, la honradez, etc. La comprensión de la naturaleza de las relaciones humanas, resolución de conflictos y capacidad de diálogo, constituyen factor importante (Barrientos, 2016). 


\section{El modelo educativo y la formación de docentes}

Los escenarios y los espacios actuales en las que se forman los nuevos maestros responden a una concepción de la educación reduccionista, los que se concretan mediante diseños curriculares centrados en un perfil profesional del egresado y un plan de estudios lineal o por asignaturas (parcelamiento del conocimiento), generalmente distribuidos durante el tiempo que dura la formación profesional. Los modelos lineales, a menudo se presentan como innovaciones con la adición de ciertos elementos modernizantes, tales como: competencias, en vez de objetivos u objetivos de conducta, etc. Responde a una concepción mecanicista del aprendizaje que genera la fragmentación del conocimiento y fomenta la pasividad del estudiante.

De acuerdo al análisis de los modelos educativos, concepción de la educación, visión, misión y valores asumidos por las instituciones de formación docente, se observa una fuerte tendencia del modelo de la escuela positivista, donde se prioriza trasmisión de información científica academicista durante toda la formación profesional del docente.

Según Carlos Tünnermann Bernheim (2008) "el modelo educativo es la concreción, en términos pedagógicos, de los paradigmas educativos que una institución profesa y que sirve de referencia para todas las funciones que cumple (docencia, investigación, extensión, vinculación y servicios), a fin de hacer realidad su proyecto educativo" (p. 14). Sin duda, si el modelo pretende promover una formación integral sobre la base de la perspectiva multinivel-multidimensión, si pretende formar estudiantes críticos, participativos, capaces de asumir su propio desarrollo autónomo, de ser creativos, disciplinados en su vocación de educadores.

El modelo educativo de una Universidad "es la filosofía educativa de la institución, a partir de la cual se desprenden metas y líneas de acción que impulsan el mejoramiento continuo de las personas y su entorno" (Farro, 2012, p. 78). Naturalmente, un modelo educativo consiste en una recopilación o síntesis de distintas teorías y enfoques pedagógicos, que orientan a los docentes en su práctica pedagógica. Cada institución educativa asume un modelo educativo de acuerdo a su misión, visión y valores institucionales las que son interiorizadas y puestas en práctica por la comunidad de aprendizaje. Por lo que, el modelo educativo se define en congruencia con lo que la institución de formación docente es (misión) y lo que aspira a ser (visión).

De manera resumida presentamos los modelos educativos que aún persisten en las instituciones educativas 


\section{Enfoque conductista}

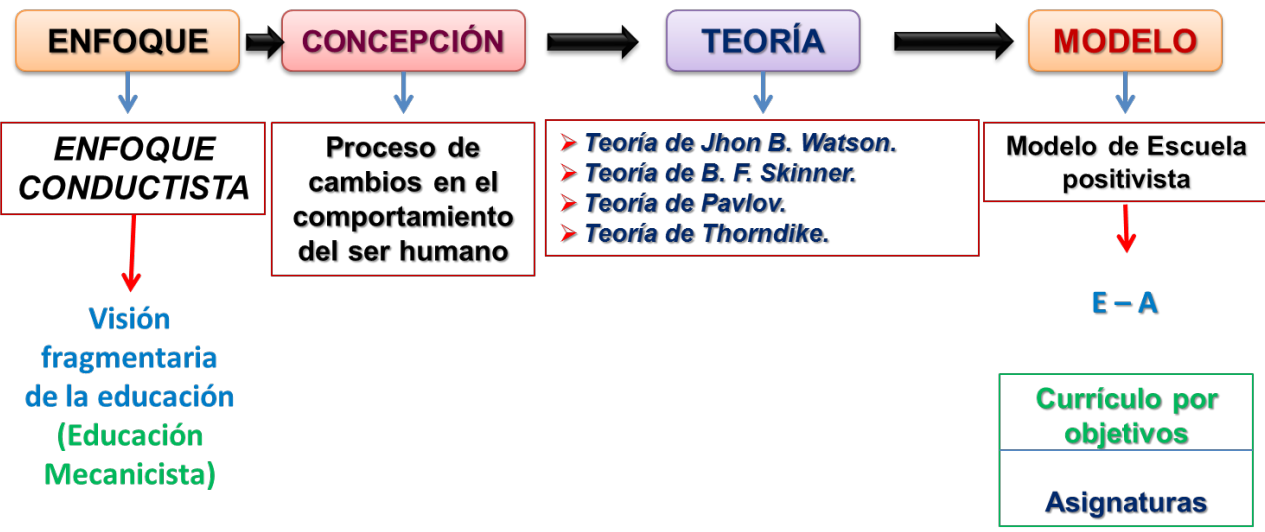

De acuerdo al enfoque conductista de la educación, el modelo de enseñanza es el condicionamiento: adiestrar-condicionar; siendo el aprendizaje como producto de una relación "Estímulo - Respuesta". El estudio del aprendizaje debe enfocarse en fenómenos observables y medibles, en la que el aprendizaje únicamente ocurre cuando se observa un cambio en el comportamiento del educando, si no hay cambio observable no hay aprendizaje. Desde esta perspectiva el aprendizaje es definido como un cambio observable en el comportamiento, los procesos internos (procesos mentales superiores) son considerados irrelevantes para el estudio del aprendizaje humano ya que estos no pueden ser medidos ni observados de manera objetiva y directa. Este enfoque pedagógico es propio de la sociedad industrial; siendo el modelo de producción el fordismo, un modelo de trabajo en cadena donde uno piensa y otros realizan sin pensar y mecánicamente lo que aquel ha pensado. En el sistema educativo, este modelo educativo promueve una sociedad consumista bajo un pensamiento convergente y la dependencia en todo aspecto de la vida de la nueva generación de la sociedad.

\section{Enfoque cognoscitivista}

\begin{tabular}{|c|c|c|c|}
\hline ENFOQUE & CONCEPCIÓN & TEORÍA & MODELO \\
\hline$\downarrow$ & $\downarrow$ & $\downarrow$ & $\downarrow$ \\
\hline $\begin{array}{l}\text { ENFOQUE } \\
\text { COGNITIVO }\end{array}$ & \multirow{2}{*}{$\begin{array}{l}\text { Proceso de } \\
\text { desarrollo de las } \\
\text { habilidades } \\
\text { cognitivas }\end{array}$} & \multirow{3}{*}{$\begin{array}{l}\text { La Teoría constructivista del } \\
\text { aprendizaje (experiencias } \\
\text { previas) de Piaget y Bruner. } \\
\text { Teoría conceptualista del } \\
\text { aprendizaje (saberes previos) } \\
\text { de Ausubel y Novak. }\end{array}$} & $\begin{array}{l}\text { Modelo de Escuela } \\
\text { positivista }\end{array}$ \\
\hline \multirow{4}{*}{$\begin{array}{l}\text { Visión } \\
\text { fragmentaria } \\
\text { de la educación } \\
\text { (Educación } \\
\text { Mecanicista) }\end{array}$} & & & $\downarrow$ \\
\hline & & & $A-E$ \\
\hline & & & $\begin{array}{l}\text { Curriculo por } \\
\text { competencias }\end{array}$ \\
\hline & & & $\begin{array}{c}\text { Áreas } \\
\text { curriculares }\end{array}$ \\
\hline
\end{tabular}


El enfoque pedagógico cognitivo, responde a un modelo de escuela positivista donde la enseñanza se subordina al aprendizaje del educando. Es más individualista (centrado en los procesos del individuo), siendo el aprendizaje como proceso cognitivo. Sin embargo el educando es un sujeto activo procesador de información. Para ello el profesor parte de los saberes y experiencias previas de los educandos, que supuestamente debe generar aprendizajes significativos. Este enfoque responde a una filosofía de la modernidad y su correspondiente cultura científico-industrial. Sin embargo, este modelo de escuela positivista responde a una visión fragmentaria de la educación, donde la sesión de aprendizaje se concreta a la trasmisión de la información académica y no para el desarrollo de las capacidades en el logro de las competencias de los educandos, a pesar de que se trabaje con un currículo por competencias.

\section{Enfoque contextual o socio-cultural}

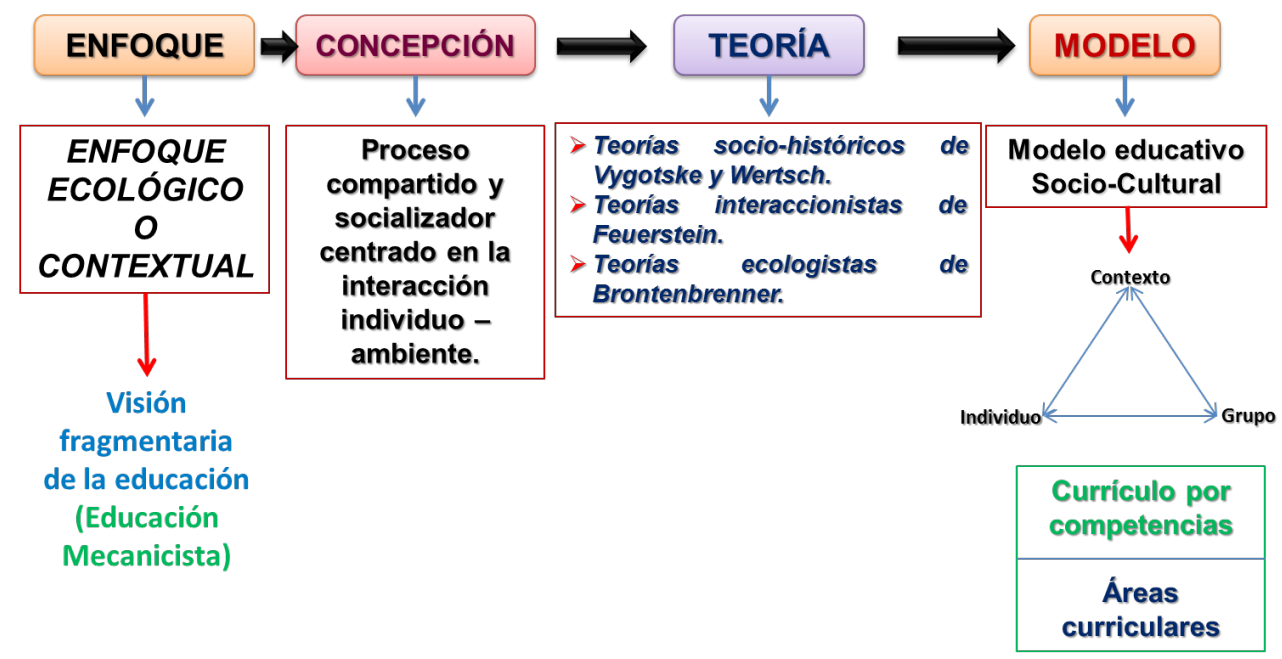

Bajo este enfoque pedagógico el modelo de enseñanza se centra en la interacción individuo-ambiente. El aprendizaje es compartido y socializador, donde se preocupa del entorno y de la vida del aula y ambos aspectos pueden y deben ser complementarios. El paradigma ecológico es más socializador (centrado en la interacción contexto-grupo-individuo y viceversa). Sin embargo, la visión de la educación es fragmentaria, ya que se centra sólo en el desarrollo de la dimensión social de la educación, descuidando el desarrollo de las dimensiones corporal, cognitivo, emocional, estético y espiritual del estudiante. 


\section{Enfoque constructivista}
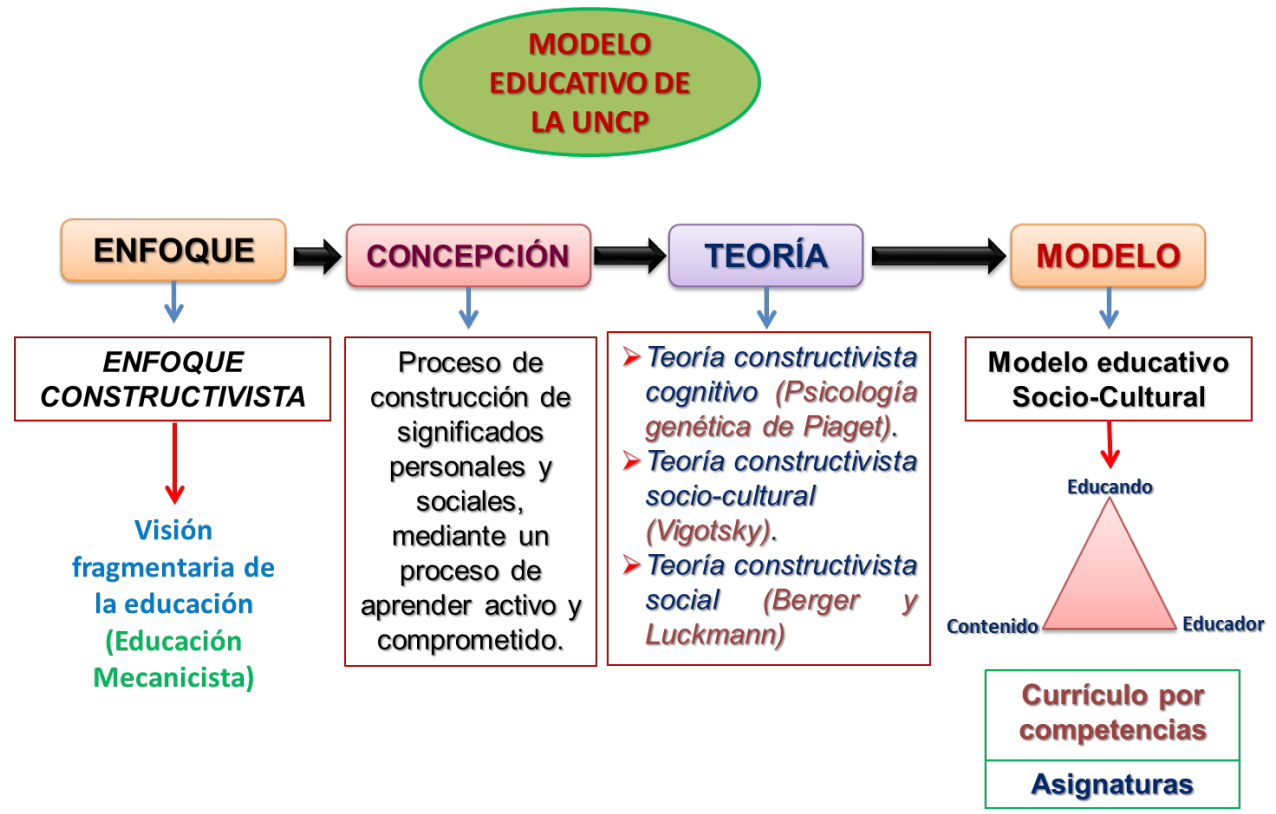

\section{Modelo educativo integral: Desafíos en formación docente del siglo XXI}

Teniendo en cuenta los nuevos escenarios, tendencias y la prospectiva educativa, la formación de nuevos docentes debe ser innovado a partir de la visión integral de la realidad educativa para comprender o entender de manera holística y tener una concepción integral de la educación. El describir y explicar el conjunto de hechos y fenómenos educativos a partir de la comprensión de la realidad, constituyen las distintas teorías que nos permiten actuar de manera adecuada en la formación integral de los estudiantes y la forma cómo asumimos esas teorías y los enfoques pedagógicos en nuestra práctica pedagógica, viene a ser el modelo educativo.

El desafío en la formación integral de los docentes para el siglo XXI, requiere replantear la visión de la educación a partir del enfoque holístico y la concepción de la educación bajo el pluralismo epistemológico, siendo los fundamentos teórico-metodológicos: la filosofía perenne, los nuevos paradigmas y los grandes pedagogos, concretando para la práctica pedagógica un modelo educativo integral con perspectiva multinivel-multidimensión, a través de un perfil humano-profesional, currículo por competencias y bajo la pedagogía del ejemplo. 


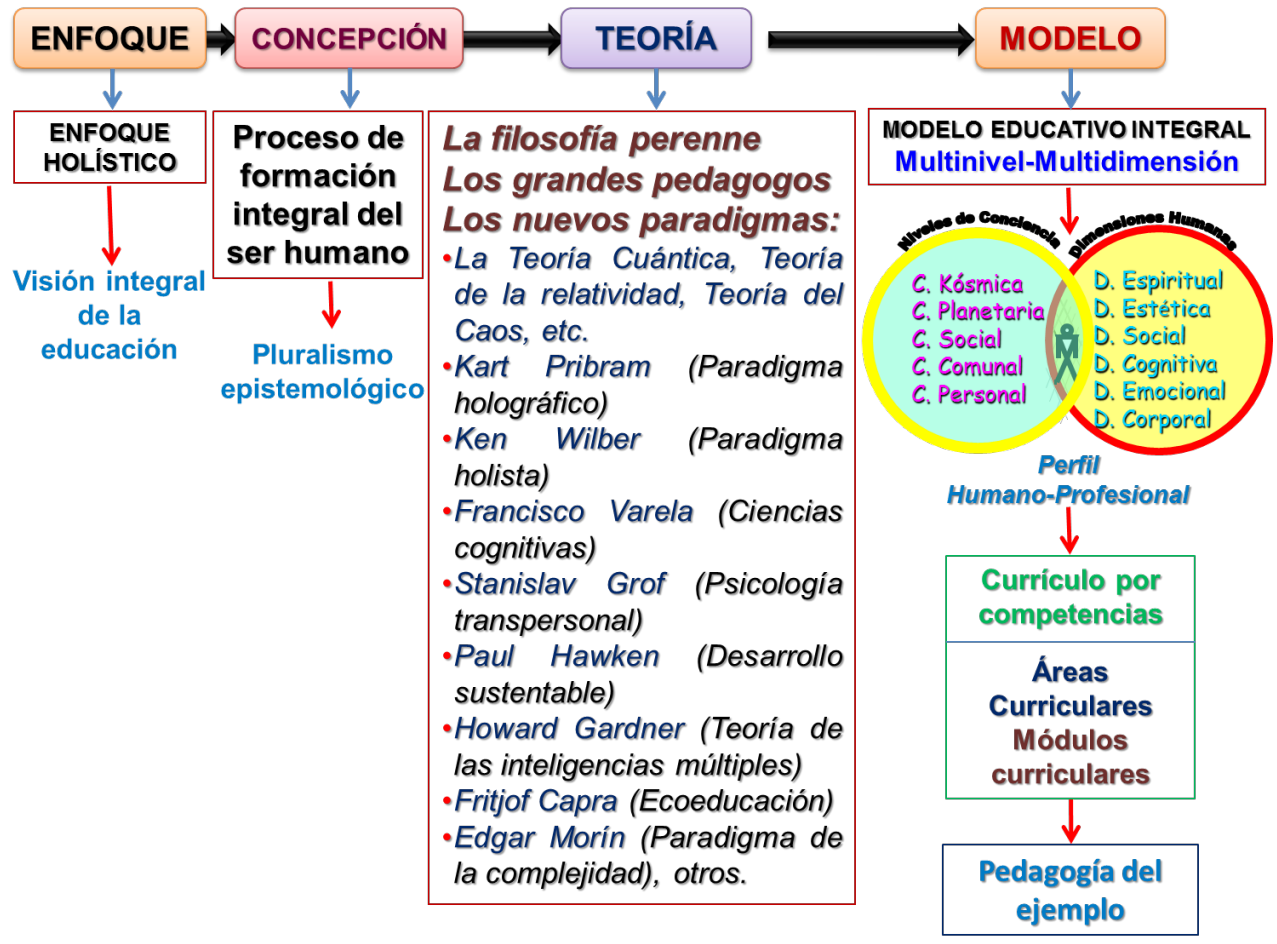

Fuente: Sistematizado por Barrientos a partir de Gallegos (2003).

El modelo educativo integral multinivel-multidimensión sistematizado de acuerdo a Ramón Gallegos Nava (2003) está basado en la diferenciación e integración de cinco niveles de totalidad educativa, cinco estadios de evolución de la conciencia y seis dimensiones de aprendizaje. Cuando estos niveles y dimensiones se integran emergen treinta regiones educativas - llamados también 30 mapas pedagógicos, desde lo más mecánico y personal (Educación mecanicista/fragmentaria) hasta lo más sutil y universal (Educación integral).

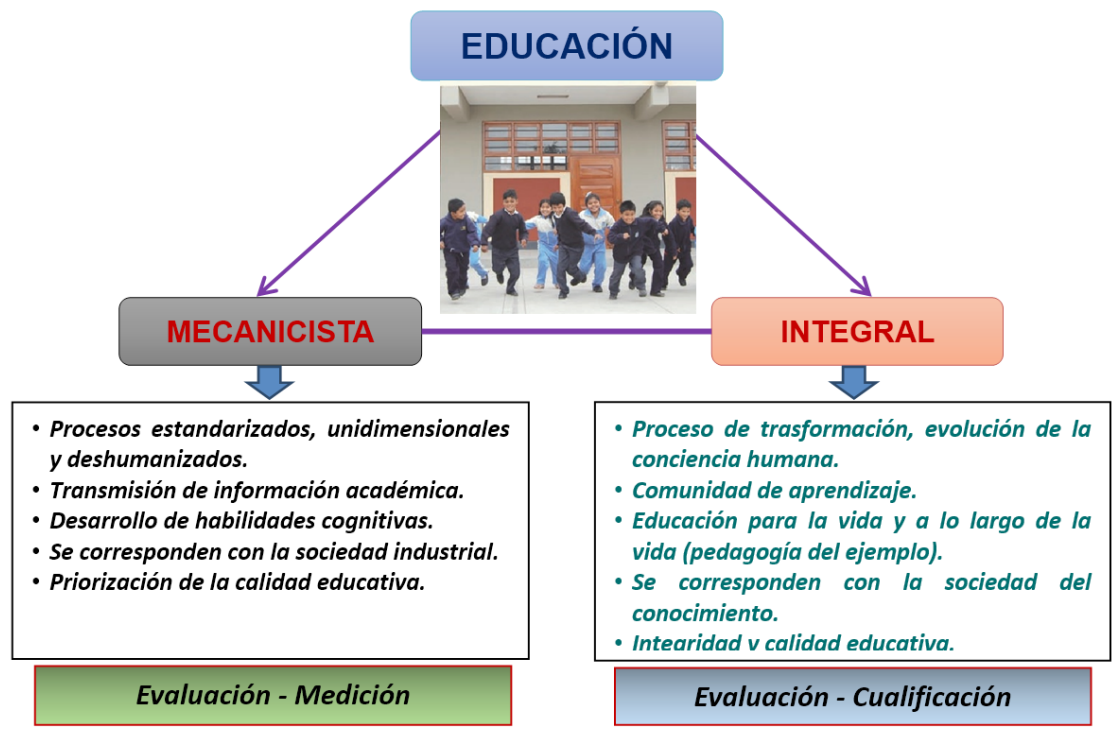


La esencia en la formación integral del docente es el desarrollo de las seis dimensiones humanas (Corporal, social, cognitivo, emocional, estético y espiritual) y los cinco niveles de conciencia (personal, comunal, social, planetaria y kósmica), lo que conduce a tener el perfil humano y profesional para actuar en la práctica pedagógica con las condiciones necesarias y adecuadas para tratar a los estudiantes como a seres humanos. Al respecto gallegos (2001) nos presenta la perspectiva multinivel-multidimensión de la educación con las siguientes características:

1. Es una visión integral de la educación que nos permite integrar las partes de una gran imagen global.

2. Un modelo integral que considera los diferentes niveles y dimensiones de la experiencia educativa y nos permite ubicar e integrar las diferentes teorías educativas del pasado y del presente.

3. Desde una perspectiva multinivel, la educación puede ser considerada en cinco niveles de totalidad, cuya naturaleza es la evolución de la conciencia.

4. La perspectiva multidimensión complementa e integra la perspectiva multinivel.

5. En todo proceso de aprendizaje, siempre están presentes seis dimensiones, ya que el estudiante es un ser integral, el aprendizaje no es un acto solo cognitivo.

6. Cuando los niveles y dimensiones se integran, emergen 30 regiones educativas, desde lo más mecánico y personal hasta lo más sutil y universal.

Sobre la base de los escenarios, tendencias y prospectiva descritos, y tomando en consideración los diferentes enfoques pedagógicos, el modelo educativo para concretar la formación humana y profesional del futuro docente debe ser un modelo educativo multinivel- multidimensión que tiene como propósito fundamental la formación holística de los estudiantes. Un nivel de organización del proceso integral de intervención educativa que exige la sociedad actual.

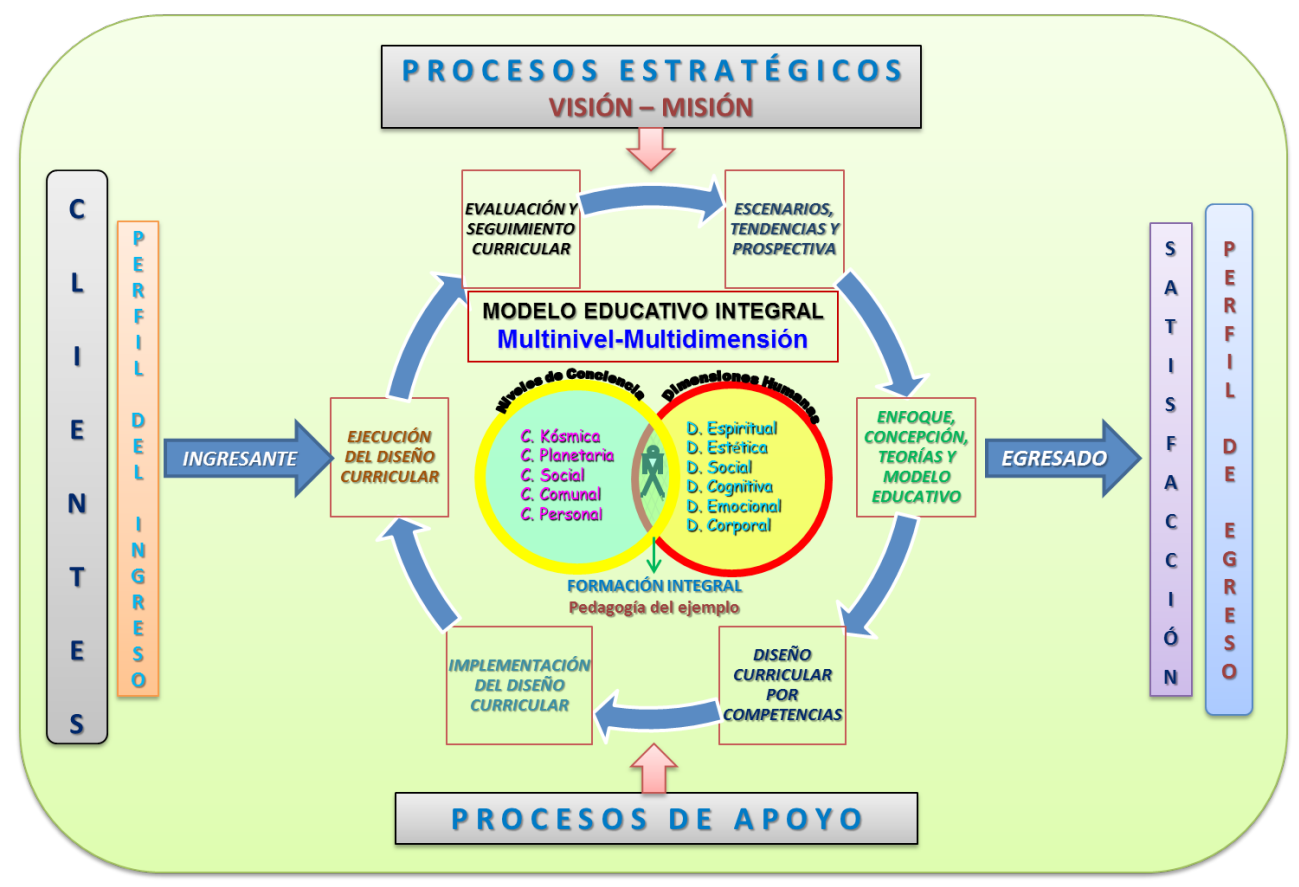


El proceso de formación integral del docente constituye cambios profundos en la conciencia del estudiante, para ello se requiere de espacios y escenarios de aprendizaje que facilite el desarrollo integral, los que constituyen la comunidad de aprendizaje (Universidad, sociedad, grupos de interés y los egresados). Las comunidades de aprendizaje son ámbitos educativos integrales, procesos colaborativos que participan de manera directa e indirecta en la formación humana y profesional de los futuros docentes.

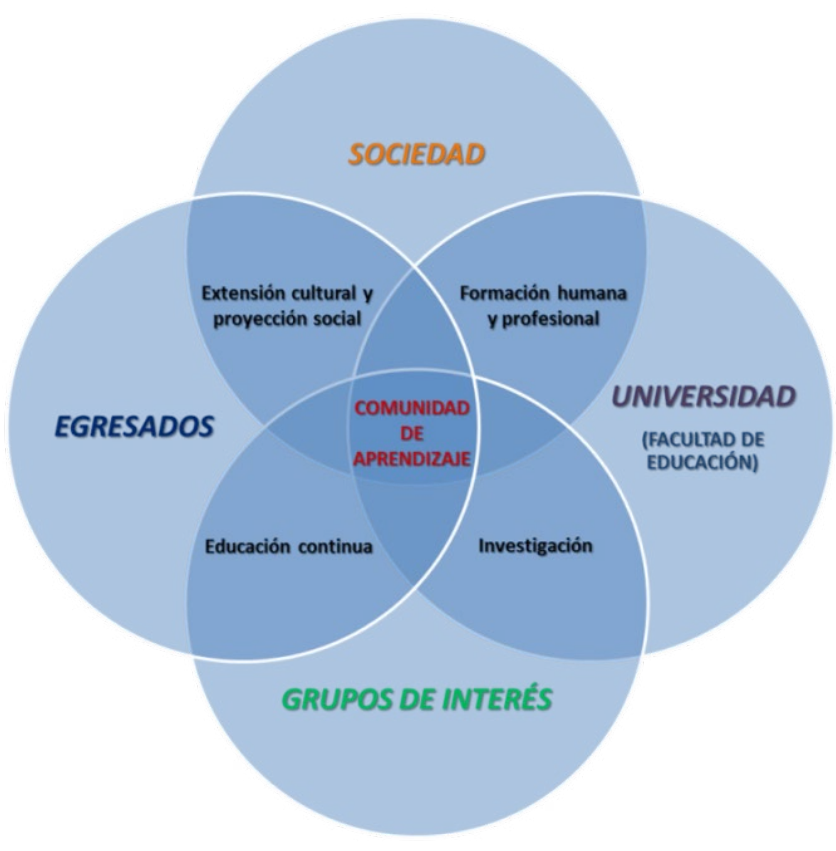

\section{Diseño curricular de formación docente por competencias}

Responder al cambio del entorno y a las exigencias de la sociedad actual, requiere innovación curricular en la formación humana y profesional del futuro docente. Asumir un diseño curricular que concrete la concepción de la educación integral de modelo educativo multinivel-multidimensión, implica el logro de competencias mediante el desarrollo de capacidades organizadas y ejecutadas por el docente que ejerce su desempeño de práctica educativa con la pedagogía del ejemplo y la afectividad en todo el proceso del aprendizaje.

Un diseño curricular por competencias para la formación integral del docente bajo el modelo educativo multinivel-multidimensión es una necesidad innegable en este siglo XXI. Su construcción requiere tener ideas claras respecto:

1. Carácter probabilístico y aproximativo de la educación y el currículo.

2. Relatividad a nivel de supuestos o presupuestos curriculares.

3. Visión totalizante e integradora del currículo de educación superior. 
4. Multifactorialidad de la vida y el mundo.

5. Transdisciplinariedad curricular.

6. Perspectiva multinivel-multidimensión de la educación y el currículo.

7. La ley de relación de las ciencias.

Todo ello implica el manejo de estrategias adecuadas en la construcción curricular que exige retos para trascender:

1. Del plano lingüístico al plano fáctico del currículo.

2. De las abstracciones metodológicas a las concretizaciones procedimentales.

3. De la metodología algorítmica a la metodología heurística.

4. Del pensamiento convergente al pensamiento divergente lateral flexible.

5. Síntesis metodológica proactiva.

6. Alternancia metodológica.

La base del conocimiento científico del diseño curricular se explica de manera diferente en función de la óptica adoptada de las teorías del modelo educativo; es por ello, "el currículo está constituido por una serie de supuestos teóricos, doctrinarios y tecnológicos, que van a posibilitar los cambios sociales y educacionales" (Canduelas, 1996, p. 22). En este contexto, el currículo constituye un medio para materializar o concretizar la concepción de la educación en el proceso de enseñanza y aprendizaje.

El trabajo curricular en las actuales circunstancias de altas contingencias sociales y naturales que vivimos, la interdisciplinariedad y la transdisciplinariedad de una percepción integral de la educación, debe ser nuestra preocupación en la materialización curricular de una clara tendencia crítico-reflexiva para que el aprendizaje de los estudiantes, sea un conjunto de experiencias vivenciadas bajo la organización y mediación del docente para el desarrollo de capacidades en el logro de las competencias humanas y profesionales del estudiante.

De acuerdo con Barrientos (2007) entre las constantes o denominadores comunes típicos de situaciones educacionales y del currículo, dentro de una visión integral de la educación, constituyen:

1. Los fenómenos histórico - sociales de los pueblos, plenamente relacionados con los fenómenos Bio-Físico-Químicos del mundo natural.

2. El plano holístico transdisciplinar del currículo, donde los sujetos, elementos y procesos del currículo tenga una interacción ontológica, de esencia, contenidos - perfiles.

3. La desaparición de las fronteras entre las disciplinas, convirtiéndose en una transdisciplinariedad, puesto que estamos en un mundo de las relaciones, no existe ciencias aisladas; todos los fenómenos de la naturaleza están relacionados con los fenómenos histórico-sociales de los pueblos.

4. La transdisciplinariedad es el constante eje, porque los problemas educacionales debe ser enfocado desde diferentes ángulos.

5. Currículo altamente contemporizado, porque constituye un compromiso entre lo deseable y lo factible.

6. La pertinencia social y altamente cualitativo.

7. Reconocimiento pleno de la ruptura epistemológica.

8. Manejo de contenidos transdisciplinares, ejes y denominadores comunes.

9. Estrategias metodológicas interactivas.

10. Evaluación integral, criterial, diferenciada y con énfasis de evaluación formativa. 
11. La práctica y la investigación pedagógica como eje orientador fundamental en la formación del docente.

En un currículo por competencias, las competencias han sido definidas y asumidas de diversas maneras:

1. Desde un simple saber hacer que pone énfasis en la conducta observable y verificable de los individuos (Enfoque conductista).

2. Un saber referido a las funciones laborales requeridas en el desempeño de una ocupación (Enfoque funcionalista).

3. El saber adquirido con la participación activa de la persona en su propio aprendizaje (Enfoque constructivista).

4. Un saber complejo que integra un saber hacer, un saber conocer y un saber ser, implicando una actuación integral de la persona para analizar y resolver problemas del contexto en distintos escenarios (Enfoque sistémico complejo).

5. Desde la visión integral de la educación la competencia es entendida como un conjunto de actos internos que guían las conductas externas (Enfoque holista); siendo la educación como proceso de trasformación, evolución de la conciencia humana.

En la construcción curricular, las bases y los fundamentos constituyen el sustento teórico en el que se basa todo diseño curricular por competencias. En este nivel se define el tipo de hombre que se pretende formar, la ubicación y posición de ése hombre en la sociedad, así como su nivel de participación en la misma, entre otros; es decir, que se define el tipo de orientación o concepción que tendrá el currículo que se pondrá en práctica. Es por ello, el currículo para ser pertinente debe sustentarse en fundamentos o referentes teóricos que va servir de sustento a la construcción curricular bajo la visión integral de la educación y los principios del currículo por competencias que responde al modelo educativo multinivel-multidimensión (Barrientos, 2007, p.80).

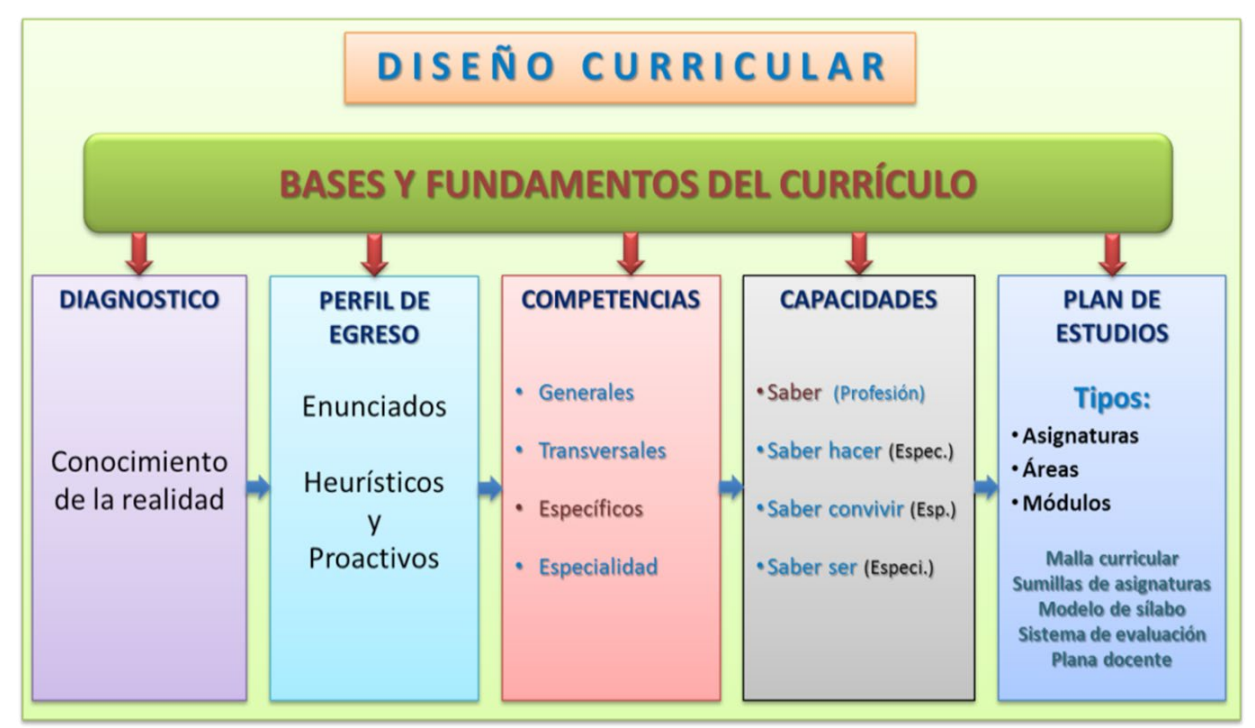




\section{Sistema de evaluación del aprendizaje}

La evaluación en un currículo por competencias constituye un proceso pedagógico inherente a la enseñanza y al aprendizaje que permite observar, recoger, analizar e interpretar información relevante acerca de las necesidades, posibilidades, dificultades y logros de aprendizaje de los estudiantes, con la finalidad de reflexionar, emitir juicios de valor y tomar decisiones pertinentes y oportunas para mejorar la enseñanza, y por ende, el aprendizaje de los estudiantes.

Se evalúa la competencia a partir de las capacidades y desempeños previstos en la programación, para lo cual es necesario formular criterios e indicadores de evaluación, a partir de ello determinar las técnicas e instrumentos de evaluación para recoger información relevante de los aprendizajes adquiridos y luego establecer los niveles de logro alcanzados por los estudiantes.

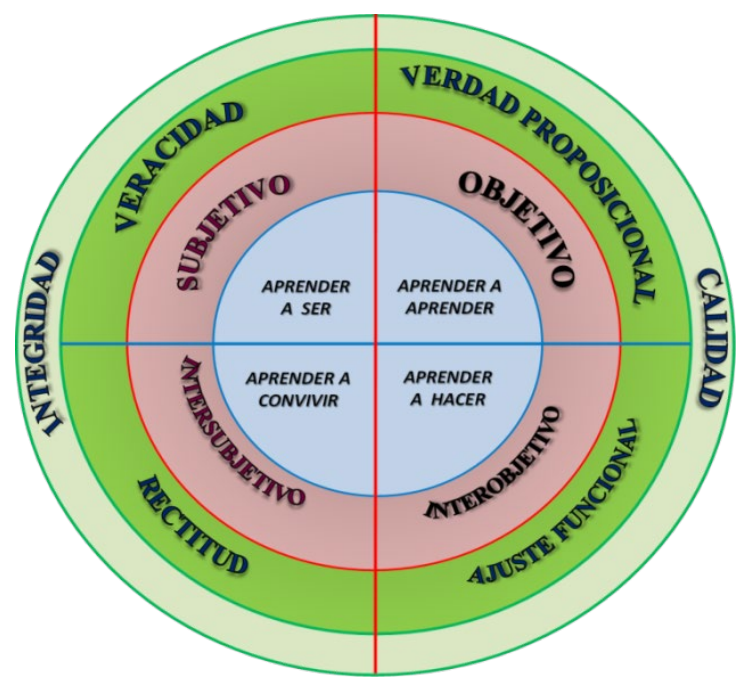

Bajo la visión integral de la educación en un currículo por competencias, la evaluación es integral, permanente y valorativo, siendo esta evaluación no desde una, sino desde cuatro formas diferentes de verificación, cada una de estas formas se corresponden con cada uno de los cuadrantes e implica un marco epistemológico diferente en el sistema de evaluación:

1. Objetivo individual (Tipo de verificación centrado en la verdad proposicional). Son medibles, empíricos y repetibles. Se observan los hechos individuales desde fuera en términos empíricos. Aprender a aprender (Conciencia científica).

2. Objetivo social (Tipo de verificación centrado en el ajuste funcional). Es un proceso de observación empírico analítica, es la típica evaluación sociológica sistémica. Interobjetivo. Aprender a hacer (Conciencia social).

3. Subjetivo individual (Tipo de verificación centrado en la veracidad subjetiva). Es un proceso de interpretación hermenéutica de eventos ubicados en estado de conciencia. El diálogo y la interpretación son las vías para conocer la interioridad y subjetividad de los sujetos. Se busca la verdad, sinceridad y honestidad. Aprender a ser (conciencia espiritual).

4. Subjetivo social (Tipo de verificación centrado en la rectitud intersubjetiva). Es un proceso de ajuste intersubjetivo al sustrato cultural. Es la típica evaluación sociológica cualitativa de comprensión cultural. Aprender a vivir juntos (Conciencia ecológica). 


\section{Conclusión}

Un modelo educativo define el tipo de orientación o concepción que tendrá el diseño curricular que se pondrá en práctica en la formación docente; porque, la concepción de la educación es un fin que intentamos realizar empleando como medio una previsión de procesos y experiencias que los estudiantes han de vivenciar; esta previsión de experiencias y procesos es el currículo.

La formación humana y profesional del docente requiere de un modelo educativo integral bajo la perspectiva multinivel-multidimensión, basado en una visión integral de la educación y el pluralismo epistemológico, dejando la formación profesional bajo la filosofía y enfoque pedagógico positivista o neopositivista de la ciencia que conduce a una visión mecanicista y fragmentaria de la formación profesional del docente.

El modelo educativo integral multinivel-multidimensión en la formación humana y profesional del docente responde a las nuevas demandas educativas de la sociedad actual que exige seres humanos integrales, inteligentes y con un profundo amor a la vida y amar la vida es amar el aprender a aprender, aprender a hacer, aprender a convivir con los demás y aprender a ser; todo ello, educado con una pedagogía del ejemplo y la afectividad, ya que el ejemplo vivenciado 
Barrientos, P. (2016). La Naturaleza de la Formación Docente. Revista Horizonte de la Ciencia, 6 (11), 169-177. Diciembre 2016 FE/UNCP. ISSN (Impreso): 2304-4330/ ISSN (En Línea): 2413-936X. http://www.uncp.edu.pe/revistas/index.php/horizontedelaciencia/about/index

Barrientos, P. (2007). Visión holista de la Educación. Hacia un aprendizaje con rostro humano. Lima: Editorial Ugraph S.A.C.

Canduelas, A. (1996). Teoría y planificación curricular. Lima: U.N.E. “Enrique G. Valle”. La Cantuta.

Farro, F. (2012). Planeamiento y gestión estratégica para universidades peruanas competentes. Modelos, Estrategias y buenas prácticas. Lima: Editorial Ugraph S.A.C.

Gallegos, R. (200o). El Espíritu de la Educación. Fundación Internacional para la Educación Holista. México.

Gallegos, R. (2001). Una Visión Integral de la Educación. Fundación Internacional para la Educación Holista. México.

Gallegos, R. (2003). Educación Holista. Fundación Internacional para la Educación Holista. México.

Gallegos, R. (2003). Comunidades de Aprendizaje. Fundación Internacional para la Educación Holista. México.

García J., López N., Rodríguez J. y Tobón S. (2008). Gestión del Currículo por Competencias. Una aproximación desde el modelo sistémico complejo. Lima: F. M. Servicios Gráficos S. A.

Peñaloza, W. (2000). El Currículo Integral. Lima: Optimice, Editores.

Tünnerman, C. (2008). Modelos Educativos y Académicos. Editorial Hispamer.

Universidad Autónoma de Yucatán (2003). Modelo Educativo y Académico, Mérida, Yucatán, México. 